again becoming audible. The probable cause of the existence of an aneurism so large as perceptibly to raise the liver, without any aneurismal bruit, may be sought in the large size of the opening into the artery; which is also, no doubt, the reason why so many thoracic aneurisms present no appreciable bruit. The subsequent appearance of a bruit must be referred to some change in the condition of the orifice, probably to its becoming partially obstructed by the "stratified fibrine" noticed there, and its temporary latency by some subsequent change or displacement of this clot.

The case of abdominal aneurism from the Birmingham General Hospital, which precedes this, presents several interesting points of comparison as well as of contrast.

\section{HOSPITAL FOR SICK CHILDREN.}

I. ABSCESS IN THE KNEE-JOINT: RECOVERY.

Under the care of Athol A. Johnson, Esq.

IHE annexed case is cited as an example of how much may be effected by patience and the expectant plan of treatment in cases of diseased joints in children, more especially when the disease does not commence in the bones, but in the surrounding soft parts. In such cases, it is, as far as our experience goes, hardly ever necessary to proceed to the resection of the joint, inasmuch as a useful (and very often a flexible) limb usually is preserved by very simple methods, which may be summed up in a few words-rest, tonics, and incisions. On the other hand, as those affections of the joints which do involve the bones in childhood usually proceed from constitutional causes, and affect the whole or a great part of the bone in question, it would follow that, in children, the operation of resection is not often indicated; and that, indeed, appears to be the opinion which gains ground more and more among those who have an opportunity of seeing much of the diseases of early life. It does, indeed, every now and then happen that circumstances prevent the long period which is necessary for the spontaneous cure being given to the case, and that an operation becomes advisable, in order to hasten its completion; but these cases are the exception, and, as a rule, our experience of the practice at this Hospital would lead us to the belief that resection will become rarer and rarer in childhood, in proportion to the advance of our knowledge of the pathology of the diseases of that period.

Alfred T., aged 2, a pale delicate looking infant, was admitted on August 21st, 1858, with great swelling of the right knee, not confined to the joint, but extending to some distance upwards and downwards. The joint was semiflexed, and any movement of the part caused great pain and violent screams. The nights were disturbed, the child was losing flesh, and the swelling was increasing in size. The skin was hot; the pulse quiet.

The knee was first perceived to be swollen in February 1858; but the child had had a fall three months previously, since which time he had appeared occasionally in pain. He was taken to another hospital, but no disease was then detected. In March he was admitted as an ont-patient at this Hospital, when the limb was placed on a splint, and cod-liver oil given. Latterly, however, it had appeared to get much worse, so that it was necessary to take him into the Hospital. He was ordered quinine mixture, beef-tea, etc., and was kept quiet in bed; but the swelling increased, the skin became red, and the pain very great. A free incision was made into the joint, and a large quantity of matter let out. This was attended with some relief, but matter reaccumulated, and it was necessary to make three other incisions into different parts of the suppurating cavity.

By this time (October 1st) the child had become extremely emaciated, restless nights and frequent attacks of diarrhwea threatening to carry it off. After the last incision, however, he began to rally, and to suffer less pain; and the operation of excision of the knee, which had been contemplated, was postponed. Under the use of quinine and iron, means being taken to prevent any reaccumulation of matter, he began to gain flesh, to lose pain, sit up a little, and piay with his toys.

On November 26th, he had been steadily improving, but there was still a good deal of discharge from all four openings. The knee was semiflexed, and could not be moved without pain. The appetite was good, and the pulse nearly natural.

At the commencement of the present year, he was attacked with erysipelas of the diseased leg, which extended from the toes upwards to the groin. Hot fomentations were applied 205 locally, and salines given. The erysipelas soon subsided, and was followed by great improvement in the condition of the joint, which diminished in size, the wounds assuming a healthy appearance. There was no pain, and the boy began to gain flesh rapidly. On February $26 \mathrm{th}$, the wounds were healed; the joint was still enlarged, but not much, and was stiff. It was, however, in good position, and no pain was produced by such force as it was thought proper to submit it to in examination. The child was now quite fat and healthy looking. He was made an outpatient, and was last seen on March 3rd. The knee was still kept on a splint, and cod.liver oil with iron was given. It seemed doubtful whether the joint were really ankylosed, but future observation will settle this point.

\section{SEBACEOUS TUMOUR CAUSING ABSORPTION AND PER-} FORATION OF THE CRANIUM.

Under the care of A. A. JoHnson, Esq.

We have already given instances in which the bones of the skull have been found more or less deficient below sebaceous tumours apparently of the ordinary kind. One of these, reported at p. 783 of the last year's volume, also occurred under Mr. Johnson's care. It is singular that another instance of this rare affection should come from the same gentleman's practice.

Mary A., an infaut six months old, was brought to $\mathbf{M r}$. Johnson on February 21st, with a small tumour, of about the size of a large pea, placed in the middle line, over the frontal bone, near the top of the forehead, and evidently a sebaceous tumour. The mother first noticed the swelling about three months previously, when it was about half the present size : it remained stationary till about a fortnight ago, when it began to increase rapidly in size. The child was apparently in good health, but was said to have become restless of late.

The tumour was cut down upon, and found to be placed in the loose cellular tissue below the tendon of the occipito-frontalis, and lodged in a well marked cup-like depression in the bone, to the periosteum covering which it was partially adherent, but especially at the bottom of the cup, where the adhesion was very firm. This was carefully dissected off; and then a small aperture or deficiency was found in the bone, at the bottom of the depression, into which the end of a probe could be passed, and where the pericranium appeared to be blended with the membranes beneath. The tumour, which was removed entire, presented nothing remarkable. The wound was brought together by strapping, and the child did well.

\section{Oritional Communications.}

\section{MEDICO-LEGAL CASE： UREMIA，OR OPIUM POISONING ?}

By James Weaver, Esq., Llandrinio.

IN the JourNaI of February 5th instant, a case is reported by Dr. Mushet, so like one which has come under my notice, that I am induced to send an account of it. I should have done so earlier, but waited for the result of the post mortem and analysis.

On February 2nd, 1859, I visited S. B, aged 67. He was pale, cold (though sitting before a fire), and very drowsy. He answered when spoken to ; but immediately lapsed into a state of sopor. The pupils were contracted; the pulse quick, small and weak. There was slight œdema of the feet and ankles.

February 2rd. I was requested to visit S. B. immediately, and was informed that he had had a fit about three hours before. I found him in bed, very pale and anxious; the eyes were wide open; the pupils contracted; the pulse small and laboured. There were no contortions of the face or limbs. He was breathing heavily, with a good deal of rattle over the chest. He answered once incoherently. The prognosis was evidently most unfavourable: the age and nature of the case not admitting of active measures. I ordered mustard plaisters to be applied to the legs, hot water to the feet, and a blister to the nape of the neck; also a drop of croton oil to be given, and followed by a cathartic mixture. He died in convulsions about four hours after my visit.

Post-mortem Examination, February 8th. The appearance of the body was natural. There was a wound of about two 
inches long, situate over the left eye, partly healed; the pupils were contracted. On opening the head, the appearance of the parts was natural. The pia mater was slightly congested; the vessels were somewhat prominent; the substance of the brain was healthy; but the vascular points were more prominent and numerous than usual. There was a small quantity of clear watery fluid in the left lateral ventricle; there was no clot, nor injury to the skull. The base of the brain was healthy. Chest. The heart was healthy, and of usual size; the lungs were pale anteriorly, but seriously congested at the base and posteriorly, with a good deal of mucus lining the bronchial tubes. There were no pleural adhesions. Abdomen. The liver was healthy; the gall bladder half full of dark thick bile. The spleen was small and firm; the stomach was distended. I removed it by ligatures, together with the intestines, for analysis. The right lidney was externally natural in size and appearance; it was slightly diseased internally. The left kidney was externally healthy; internally it was diseased, with some fat in the pelvis and surrounding parts. There was no fluid in the peritoneum; and the other organs were healthy.

In giving evidence before the coroner, on February 11th, I could not swear whether this man died of uræmia, or of poison by opium; I was not prepared to say for certain, that the amount of kidney disease found after death was sufficient to cause the former ; and, as he had lived on very bad terms with his wife, and the neighbours openly said she had poisoned him, I felt, in common fairness to the woman, that an analysis was necessary. The inquest was consequently adjourned to February 25th; and in the meantime a gentleman made an analysis of the contents of stomach and bowels. His evidence proved the non-existence of opium, and a verdict was returned accordingly, but against the wishes of some of the jury, who wanted one nothing short of manslaughter.

RFMaris. This case shews the great necessity of coroners courts of inquiry; for had not a post mortem examination and analysis taken place, the man's wife must to the end of her days have been content to bear the stigma of having poisoned her husband. The symptoms equally answered to those of opiun-poisoning or uramic disease.

\section{THE NEW TREATMENT OF WATERY EYE (EPIPHORA) : WITH ILLUS'TRATIVE CASES.}

By Janes Vose Solomon, Esq., F.R.C.S., Surgeon to the Birmingliam Eye Infirmary.

Whix the efferent lucrymal canals become either narrowed, strictured, or otherwise obstructed in any part of their course, tears collect at the inner corner of the eye, and the condition technically termed epiphora, stillicidium lacrymarum, or, in simple language, watery eye, exists.

If the lining membrane of the lacrymal sac becomes irritated, either from distension of the tears or some constitutional derangement, mucus or pus, one or both, is secreted and, unless well diluted by the tears, its escape by the natural passages is retarded ; consequently the sac is prone to become dilated, and generally remains so until relieved by the formation of an abscess, which, if followed by a permanent fistulous aperture, produces a natural cure, though at the expense of much annoyance to the patient.

Denudation of the periosteum of the bony canal I have discovered in a few instances where the secretions have been pent up in the sac, or have obtained only a tardy and imperfect vent.

A carious state of the canal is, according to my experience, a disease of rare occurrence.

The Seat of Stricture in the lid-canals has been found, in a few instances, to be near their middle; in by far the greater number, close to the tear-sac. Lower down, a frequent site is at about the point where the latter merges into the nasal duct, also at the terminal extremity of the duct.

The cases that demand much patience on the part of the surgeon, and faith in the new method, are those of adults atfected with old standing epiphora, and in whom the lid.canal is strictured close to the sac; the remainder of the excretory apparatus being so much contracted, probably from functional inactivity, that a No. 4 probe, when inserted into the duct, gives the impression of being firmly wedged.

Duringr the eleven years I have been officially connected with the Birmingham Eye Infirmary, two cases only of epiphora, which were referrible to the presence of a nasal polypus, have come under my care. The symptoms in one of them simulated those of an incipient tear-abscess.

In some of the instances where the sac has suppurated before coming under surgical observation, considerable mischief has been done to the membranous walls of the nasal duct. This condition is discoverable by the freedom with which the probe may be moved backwards and forwards on the floor of the nose. The instrument is not tightly pressed against the brow, as in the healthy subject, but has a tendency to fall forwards. The abscess appears in some cases to soften down and destroy the stricture.

'The mere fact, however, of the patient having been able, prior to the occurrence of suppuration, to press the contents of the distended sac into the nostril, is not decisive proof of the absence of stricture.

The treatment by probes passed through a slit punctum and canaliculus affords the means of ascertaining the gradual restoration of the duct to its natural dimensions. (Case virr.)

No age is exempted from epiphora. In a young gentleman of nine years, who was recently under my care, it had existed since his birth. I found the left lower canaliculus closely strictured at its sac extremity, which required division with the cannula-lancet before a probe could be made to enter the sac.

Dr. Mackenzie considers the congenital affection to be referrible to an imperfect development of the excreting canalsan opinion which derives support from the subsidence of the epiphora, in certain cases, about the age of puberty. The hereditary nature of watery eye has not been, I believe, noticed by authors, and is therefore probably of rare occurrence. I am acquainted with a family in which three of five brothers were affected in childhood, and who are still annoyed by it when exposed to the wind, though now past the meridian of life. One of them has seven sons, five of whom have manifested a similar disorder of the excreting tear-functions.

Treatment. The surgical expedients that have been devised for the relief of epiphorn are numerous, and evince much ingenuity. Those that have proved the most beneficial are based upon the principle of mechanical dilatation; some likewise affording relief, as Mackenzie and Vidal suggest, by exciting capillary attraction. In certain methods, the instruments employed have been introduced by the natural, in others by artificial openings.

J. L. Petit was, I believe, the surgeon who first clerised the plan of opening the sac through the integument which covers it, and then inserting a wax bougie into the duct.

Beer gradually dilated the duct with catgut bougies, of various sizes, which he introduced after the manner of J.L. Petit ; but, when a tear-fistula existed, he availed himself of it, enlarging the aperture when it was necessary, as $a$ means of ingress. As soon as the passages were believed to be free, the bougie was with. drawn, and measures taken to heal the wound. The chief objections to this plan are, that it treats a part only of the excreting apparatus; in the event of failure, it obliges the infliction of a fresh wound (i.e., supposing the surgeon has succeeded in closing the first one, which was prone to become fistulous), with a repetition of the whole process ab initio: moreover, it is by no means easy, as I know from experience, to hit upon the direction of a much contorted canal, when the probe is introduced from the outside of the lid. It embodies, however, a very important principle-gradual dilatation of the stricture, when such existed in the path along which the bougie travelled.

The methods of Petit and Beer, there can be no doubt, suggested the employment of the silver nail-headed style. The inconveniences attendant upon it have been represented by Mr. Bowman and others; and, as he graphically describes some of the ill consequences which had led me, previously to an acquaintance with his method of treating lacrymal obstructions, to almost entirely abandon the use of the style among the labouring poor, I quote his words.

"'The insertion of a style or tube through an opening made in front of the sac, below the tendo oculi, has been the last and common resource in obstinate cases, where the disease has worn out the patience of the sufferer and the surceon. It is an expedient so oljectionable in itself, as never to be resorted to till the last moment. It is unsightly, especially in females; it is painful, and the patient has constantly to wipe away the discharge escaping by the orifice. The puncture made to admit the style is also frequently followed by the formation of an abscess outside the sac, spreading under the orbicularis muscle, and apt to grow into that serious complication, a sinuous ulcer, 